\title{
Layered AMI Architecture for Various Grid Topologies and Communication Technologies
}

\author{
Ivan Petruševski, Graduate Student Member, IEEE, Aleksandar Rakić, Member, IEEE, \\ and Ivan Popović
}

\begin{abstract}
Advanced metering infrastructure (AMI) system, proposed in this paper, is capable of fulfilling all smart metering functionalities in real-time. The novel Local Metering Concentrator (LMC) layer is introduced as the set of independent embedded system components, allowing distributed architecture implementation. Two main data flows can be defined as command request processing and meter data acquisition. A command request, initiated by some of the top layer applications, is processed and forwarded through the system to the LMC layer, where the request is executed and the status is sent as a reply. The data and event acquisition is performed as an automatic function of the LMC layer. The collected data is forwarded to the system as soon as it is downloaded from the meters. The results of more than oneyear exploitation of the installed pilot system are presented, emphasizing the real-time load profile data acquisition, allowing the distribution system operators (DSO) and the end consumer to be actively involved in energy saving. The introduced architecture enables load profiling, energy diagnostic and easy integration with any meter data management system.
\end{abstract}

Keywords - Advanced metering infrastructure, power line communication, smart grid, smart meters.

\section{INTRODUCTION}

$\mathrm{T}$ HE set of AMI technologies provides connection between DSO and energy consumers, allowing participations of concerned parties in energy monitoring, profiling, management and saving [1-3]. The road-map of the smart grid concept emphasizes the need for real-time smart metering functionality within the AMI. Since load control, profiling and energy saving are targeted by smart metering concept, the real-time information transfer will contribute to essential benefits, individual as well as environmental, by providing support for real-time consumer side applications and energy management system

Paper received July 18, 2015; revised April 29, 2016; accepted May 3, 2016. Date of publication July 20, 2016. The associate editor coordinating the review of this manuscript and approving it for publication was Prof. Vujo Drndarević.

This paper is a revised and expanded version of the paper that received the "Ilija Stojanovic" award for the best scientific paper presented at the 22nd Telecommunications Forum TELFOR 2014 [13].

Ivan Petruševski is a $\mathrm{PhD}$ student at the Signals and Systems Department, School of Electrical Engineering, University of Belgrade, Serbia. He is with Unit-EM Ltd. Malta (ivan.d.petrusevski@gmail.com).

Aleksandar Rakić is Assistant Professor at the Signals and Systems

Department, School of Electrical Engineering, University of Belgrade, Serbia (rakic@etf.rs).

Ivan Popović is Assistant Professor at the Department of Electronics, School of Electrical Engineering, University of Belgrade, Serbia (popovici@el.etf.rs) services [4]-[6].

Based on the available experiences, one of the main deficiencies of the traditional Smart Metering Systems is the communication system between the data concentrator and electrical meter, due to a large amount of information data needed to be transferred and time consuming communication protocols. The traditional system is usually based on a proprietary communication mechanism over communication interfaces with a limited performance both in throughput and reliability, e.g. GPRS, narrow-band power line communication (N-PLC), etc. [7]. Due to mentioned technical limits, existing AMI implementation concepts provide only a basic smart metering functionality and metering data collection usually not more often than once per day. The aim of this paper is to overcome the deficiencies of traditional AMI concept by introducing a novel architecture, capable of real-time data transfer, interoperability (support for multi-vendor electrical meters), and open to implementation of various modern communication technologies [8]-[12].

In addition to the previously presented architectural overview in [13], this paper gives more details on the structure, operation and interaction between the layers of the proposed architecture, while the real-time system performance is further analyzed for the more complex system topology, including two separate transformer station areas with distinctive grid topologies. It will be shown that the system is capable of delivering 15 -minute load profile data from all the connected electrical meters within the next 15-minute interval, i.e. to provide the actual real-time performance for the data acquisition. Since there are no consequences on the system operation in the case of data loss due to a persistent communication failure and/or power outages, the system performance is regarded as the soft realtime.

The rest of the paper is organized as follows. The overview of proposed AMI architecture is presented in Section II. Section III addresses the implementation of the proposed AMI architecture in three standard power grid topologies. The following Section IV gives a description of the basic functionalities at various layers, i.e. Meter Data Collection (MDC), Transformer Station data Concentrator (TSC) and LMC. The overview of the pilot project, used for the verification of real-time characteristics, is given in Section V. Results are presented and discussed within the Section VI. Section VII presents the final conclusion and the directions of future work. 


\section{PROPOSED AMI ARCHITECTURE}

The proposed layered AMI architecture, presented in Fig. 1, introduces distributed LMC-TSC embedded implementation of AMI technologies including broadband communication at transformer station level. LMC module implementation supports standard point to point and multidrop communication with both traditional electrical meters and smart meters [14]. Thus, electrical meters' vendor specific communication protocols are integrated within LMC communication stack providing support for uniform abstraction of metering interface. Therefore, TSC-LMC communication mechanism is not burdened by vendor specific electrical meter properties, as well as time demanding communication with electrical meter $[15,16]$. Thus, TSC-LMC communication is simplified and the network throughput is significantly reduced.

The hardware of the LMC supports several communication interfaces intended for communication with meters, such as UART, RS-485 or RS-232. If requested, LMC can easily be integrated within the electrical meter itself in the form of the smart communication module, providing even wireless radio communication like ZigBee, wireless M-Bus or Z-Wave. The proposed concept can be easily extended for the communication with gas meters, water meters, heating meters or similar devices. Two main broadband communication technologies that LMC can implement toward upper layer (TSC) are broadband power line (B-PLC) and GPRS/EDGE/3G/HSDPA communication. B-PLC communication is a technology where a modulated signal in spectrum $2-32 \mathrm{MHz}$ is injected into the low voltage or middle voltage power lines, providing the IP oriented communication up to $200 \mathrm{Mbit} / \mathrm{s}$ [17], [18].

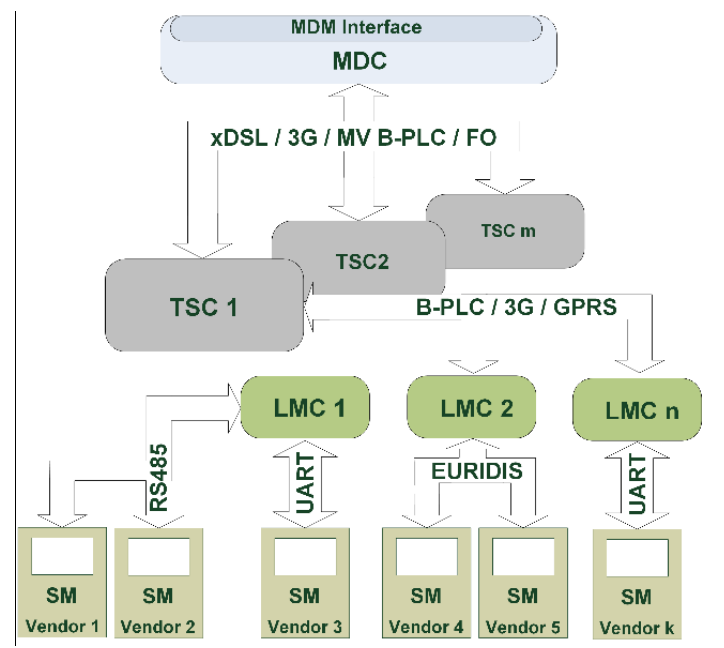

Fig. 1. The novel AMI architecture overview.

The TSC layer provides data acquisition, data transfer, command processing and other smart metering functions on the level of transformer station area. Distribution of the functionalities between the LMC and TSC layer provides robust and reliable communication, with data backup on all levels and a real-time system response.

The top layer in AMI architecture, Meter Data Collection layer (MDC) is a service oriented architecture (SOA) software solution aimed for meter data collection, data verification and correction, data storage and command processing [19, 20]. It is responsible for all data and command transfers with TSCs. MDC provides Web Service based interfaces for integration with Meter Data Management (MDM) system, network services, smart grid technologies or other enterprise systems [21].

\section{SUPPORTED AMI TOPOLOGIES}

Structure and topology of the power grid depends on many factors, such as geographical topology population density and even on diverse regulations in different countries [2], [15]. However, three typical topology types, shown in Fig. 2, are the most common ones:

- Urban topology - representing the structure where more meters are concentrated in one place, usually in a metering cabinet. This topology is typical for areas with high buildings and skyscrapers.

- Rural topology - representing the structure where every meter is in its own metering cabinet, and the distance between two meters is not substantial. This topology can be found in areas with family houses, where the population density is mild and the houses are near to each other.

- Island topology - representing the structure where every meter is in its own metering cabinet, and the distance between two adjacent meters is substantial. This topology is typical for low population density areas, like mountain villages.

(a)

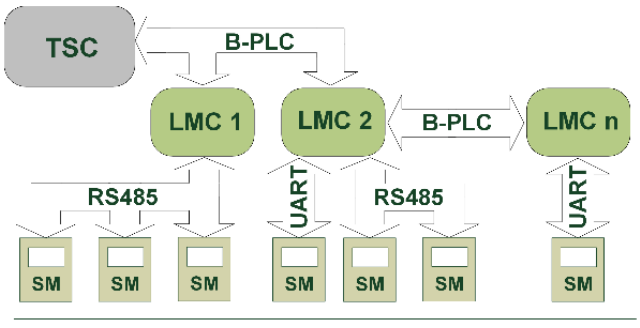

(b)

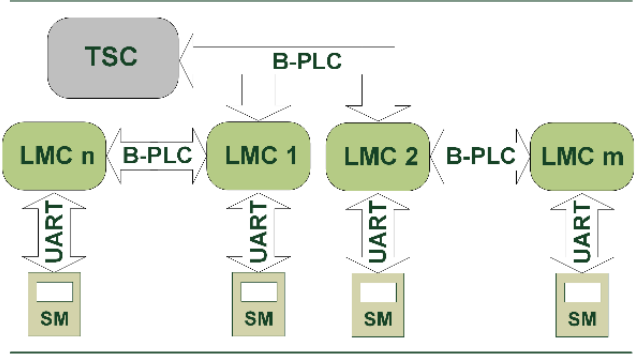

(c)

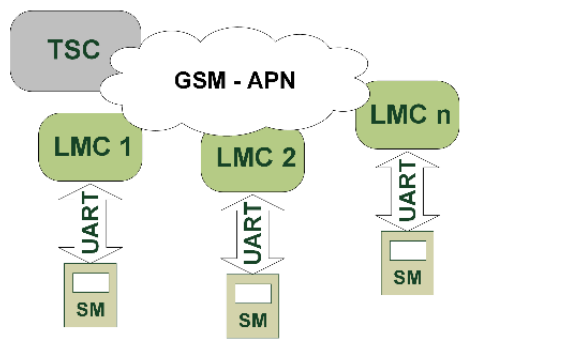

Fig. 2. Supported AMI topologies, where (a), (b) and (c) represent system architecture for urban, rural and island topology, respectively.

Regardless of topology, the novel architecture with its advanced LMC layer has an important role and achieves 
benefits for real-time Smart Metering System. Mostly used N-PLC transparent modems (one modem per one meter) are replaced with B-PLC LMC (local meter concentrator with distributed functions). Additionally, in urban topology where meters are concentrated in one metering cabinet, LMC can serve a group of meters connected to a local communication bus like RS485. Rural topology specifics, represented by the need for a significant number of repeating nodes in line between TSC and meters, are supported by LMCs automatic data repeating and correction functions that could not be implemented in any standard AMI architecture based on a narrow band transparent modem. And the last, island topology, typical for areas like mountain villages where network coverage for data traffic is not always reliable, is part of AMI system where the distribution of smart metering functions to a local level has expressed benefits in reliability and enhancement of system response dynamics.

\section{BASIC ARCHITECTURE}

The implementation concept of system components is based on SOA, which allows a high level of system scalability, interoperability and flexibility for the integration of system services and functions. Three distinct layers in the hardware architecture, MDC, TSC and LMC layer, are presented as separate blocks in Fig. 3, where each layer encapsulates the functionalities and network services in relation to the specific layer physical location in the AMI system.

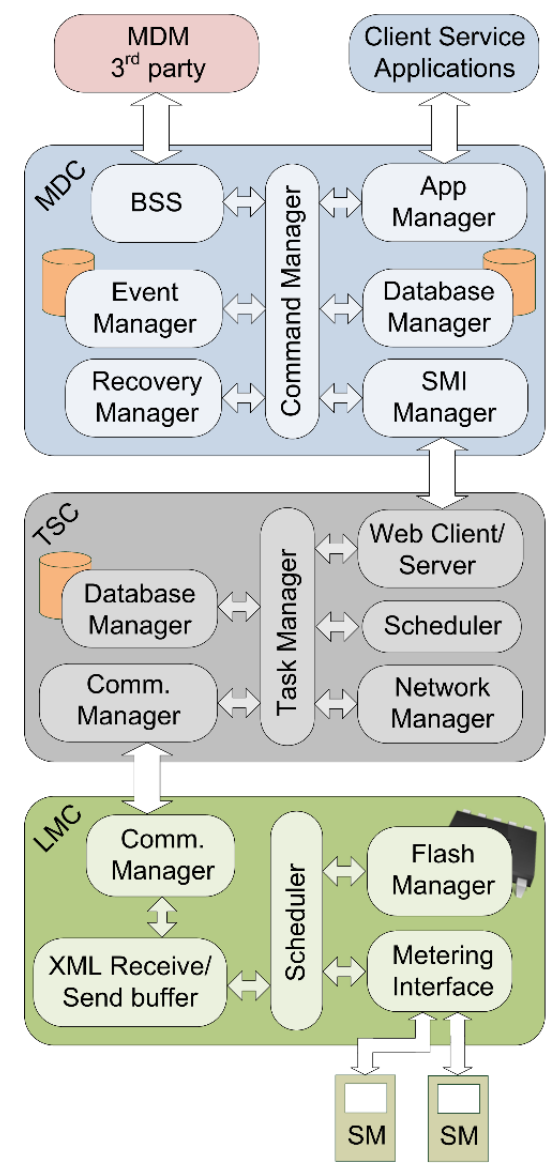

Fig. 3. Basic MDC, TSC and LMC architecture.
Although SOA enables flexibility in system topology regarding the distributed realization of the system services, the most common implementation scenario expects MDC software installed on dedicated servers in the data center, TSC inside the transformer station, while LMC could be installed inside a meter, as a meter module, or as a standalone device near the electricity meters.

The main role of the MDC layer is to process commands and collect data from all TSCs in the system, regardless of the data concentrator vendors. The Simple Object Access Protocol Web Services (SOAP-WS) are adopted, as the standard web-based connectivity solution, and it is used as the common interface for the communication between the MDC's software modules and other AMI layers. TSC is a SOA based embedded subsystem which utilizes SOAP-WS communication toward MDC layer and UDP client-server based communication toward and from LMCs. In order to support efficient but reliable message exchange, TSC-LMC communication uses an acknowledgement schema for the implementation of the automatic repeat request (ARQ) method as the error control mechanism. LMC firmware is made according to POSIX standards with several parallel threads dealing with communication with meter, providing communication channel and data exchange with TSC and storing data in a local memory.

Command Manager, as the central module of the MDC layer, is providing the connectivity between other modules, taking into consideration the task priorities and the system elements availability. Periodical data and event collection, and command processing is the main task of the SMI Manager module. Depending on the system settings, this module takes the new data on every 15 minutes and events on 5 minutes, from all TSCs in the system. The downloaded data are validated and stored into the main system database by the Database Manager module. A similar procedure is performed in the Event Manager, whose role is to store the new events, as well as to forward the event notification, according to the defined priorities, to Client Service Applications and $3^{\text {rd }}$ party MDM system, in order to notify the system operator on any tampering events or system actions and errors. Client Service Applications are dedicated applications for operating and monitoring the total system, allowing to operate the system, with its full functionality, without the integration with MDM system. The system entry point for all Client Applications is App Manager, which filters the users' actions, according to its credentials and privileges, and processes the requested tasks. One of the Client Applications is DSO Manager, whose screenshot presenting the load profile data for one of the meters, is shown in Fig. 4.

The user-friendly graphical user interface allows a very simple system data overview or meter parameter settings. MDM as a $3^{\text {rd }}$ party system, or any other external application, can be integrated with the proposed system through the BSS Manager module, which is designed as the integration platform supporting different service oriented communications and protocols, as well as the integration through database or Secure FTP Server. For the purpose of system recovery from any failure, and users' activity control, all actions are logged by the Recovery Manager. 


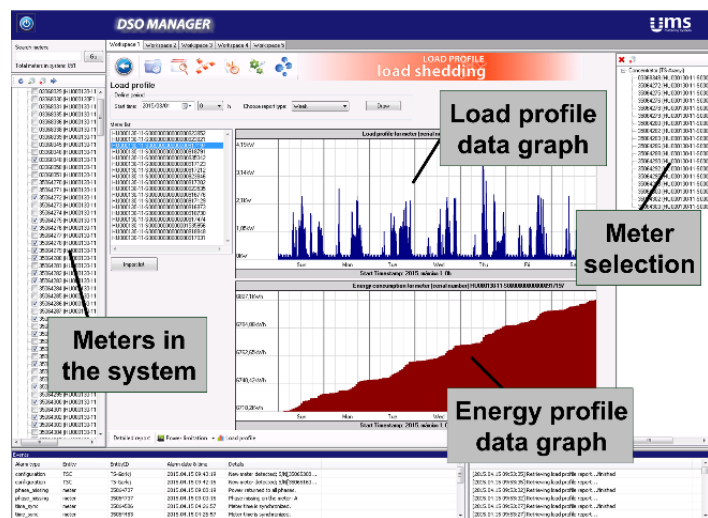

Fig. 4. DSO Manager Client Service application, presenting weekly load profile data for a single meter.

On the TSC level, the core module is Task manager, whose primary role is to process, forward and store all requests, data and events received from $\mathrm{MDC}$ and from all LMCs as well. Web Client/Server module realizes the SOAP-WS, ensuring easy integration with MDC or any other platform. Network Manager monitors and controls complete data traffic in the transformer station area which is realized by the Communication Manager. All data downloaded from meters, events, requests and TSC statuses are verified and stored into the database by the Database Manager module. As a part of TSC's advanced function, Scheduler module provides the ability of scheduling the time of execution and priority settings for various smart metering functions. All smart metering functionality on the MDC layer is also available on TSC and LMC layer making the system very robust to communication failures or other possible malfunctions.

The functionality of the LMC is distributed to three main modules: Communication Manager, Scheduler and Metering Interface. Communication Manager is responsible for reliable TSC-LMC communication on the LMC side. Intra-LMC communication between Communication Manager and Scheduler is performed over XML send and receive queues. Even though the TSC-LMC communication is UDP client-server based, the payload data is transferred in XML format and prepared for the further web-based communication supported by the entire system. The core of the LMC autonomous functionality is contained within the Scheduler module. Scheduler initiates command execution and data extraction by calling the Metering Interface (online metering data) or Flash Manager (recorded data). Two main data-flows can be defined in the system. The command request is initiated by the Client Service Applications or any $3^{\text {rd }}$ party application, then processed by the system and transferred to the LMC. Since both TSC and LMC layer contain the list of subordinate system components, the broadcast commands are translated to the sequence of the command requests to the individual listed components. The reply to the given request is sent back to the initiating instance upon the command execution. The meter data and events acquisition is the periodic scheduled action on the level of the LMC, which automatically forwards the data to the system as soon as it is downloaded from the meters.

\section{PILOT PROJECT SYSTEM OVERVIEW}

The proposed concept of AMI architecture is implemented within the pilot project, where relevant parts of the system infrastructure are presented in Fig. 5. The field results for the period of more than one year have shown a significant performance improvement compared to the traditional smart metering systems. In general, a novel AMI architecture enables real-time system operation regarding load profile data acquisition as a primary system functionality. Reliability of the system operation is also improved since in case of 15-minute load profile data acquisition, the successful network transport of metering data to the TSC stage is greater than $99 \%$. Therefore, one can conclude that the proposed architecture enables a reliable real-time information data flow between the proposed AMI system components. In addition, a novel AMI architecture enables simple integration of other realtime services and functionalities addressed by different technologies inside a smart grid concept.

Data presented in this section are obtained from two transformer station areas, which are the parts of the smart metering pilot project with a total of 851 meters. The first transformer station (TSC1) is predominantly an urban topology type area, containing 448 meters covered by 61 LMCs. Except for 12 meters that are individually connected to a $\mathrm{LMC}$, the rest of the meters are connected to a single LMC in groups of 6 to 10 meters. A mainly rural topology type is covered in the second transformer station area (TSC2), where 403 meters are integrated with 323 LMCs. In this area the majority of meters are connected individually to a LMC, while a smaller number of meters are connected in groups of 2 to 4 to a single LMC. The main communication channel, between TSC and LMC is realized with B-PLC technology, while TSCs are connected to MDC by ADSL lines.

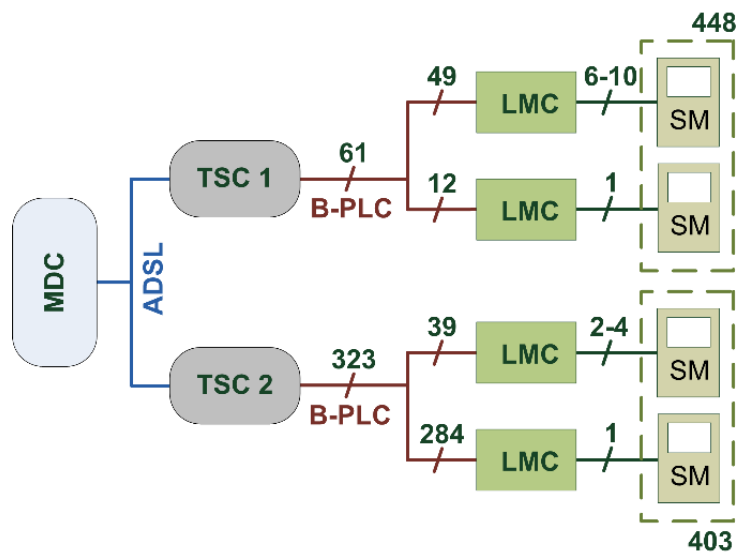

Fig. 5. Installed system infrastructure.

Datasets downloaded from the meters can be different and defined by the needs of the system operator. An XML based data structure provides a vast ability of changing the data and the data structure that should be transported. Any data managing in either of AMI system levels are documented and properly time-stamped. The structure of the XML contains unique task identification attributes and timestamps related to every layer involved in the particular command request processing or data acquisition procedure. The payload meter data in this pilot project, regarding 15- 
minute data downloaded from the smart meters (SM), contain the four tariff registers values of active energy, reactive energy registers values in four quadrants and average active power based on a 15-minute period, as well as the timestamp generated by the meter.

\section{RESULTS}

Measurement results for the availability of acquired 15-minute load-profile data at TSC layer for separate transformer station areas are presented in Figs. 6 and 7. Histograms in both figures include information on datasets for one-month period. Data stored on TSC layer can be considered as data introduced into the AMI system, since the time requirements for the TSC-MDC data transfer, via SOAP-WS, are substantially smaller than presented ones.

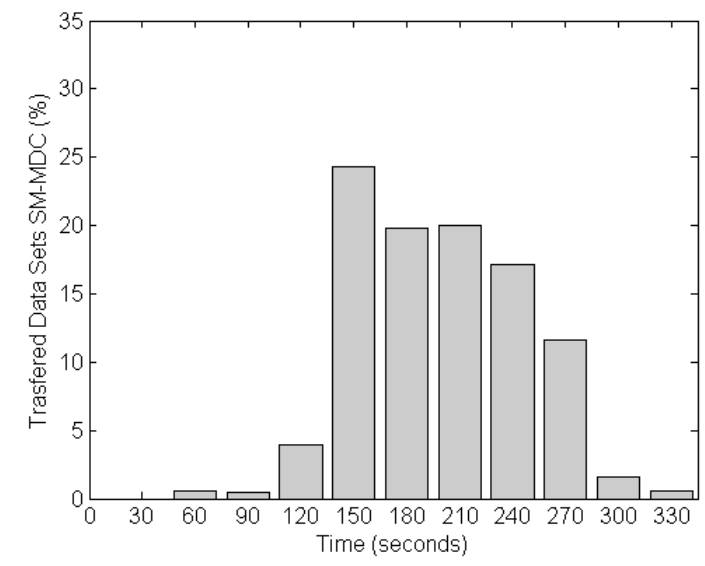

Fig. 6. Overall response time for all electrical meters in transformer station area TSC 1 , in respect to scheduled 15-minute time interval.

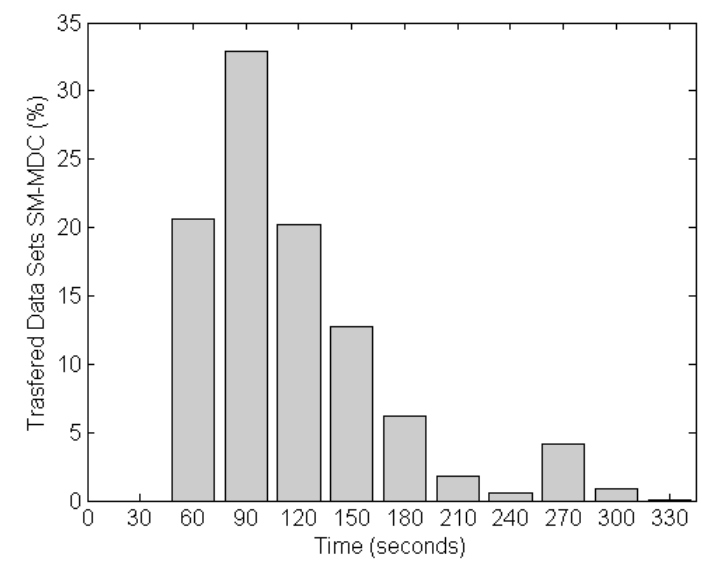

Fig. 7. Overall response time for all electrical meters in transformer station area TSC 2, in respect to scheduled

15-minute time interval.

Observing the results in Fig. 6, obtained from TSC1 (448 electrical meters), one can notice that all 15-minute datasets are downloaded in the first five-minute period, starting from the moment when a meter generates the dataset in its internal memory. The results in Fig. 7, for TSC2 (403 meters), show that time needed for downloading of majority of 15-minute datasets is less than 3 minutes, and not exceeding 5 minutes. In case of both transformer station areas, all datasets are downloaded and stored on the TSC level within a 5-minute period, but with different dynamics. Different number of installed LMCs and the fact that the LMC-SM communication is more time demanding than LMC-TSC communication, are the reasons of different dynamics of data collection in these two transformer station areas. In case of urban type transformer station area (TSC1), where most of the LMCs have 6 to 10 meters connected, more time is needed for LMC to download data from meters, but once the data is collected, data transfer to TSC is faster due to a lower number of LMCs. In the rural type transformer station (TSC2), where most of the LMCs are connected with only one meter, data transfer to TSC starts within the first minute, but the LMC-TSC total communication lasts longer because of the large number of LMCs implemented in this part of the B-PLC network. Taking into consideration that new datasets are created on meters every 15 minutes, this proves the real-time operation performance of the overall system.

Additional verification tests were performed in order to check system response time. The request for reading of current values of voltages, currents and powers was sent from the DSO Manager Client application of the MDC layer to all meters in both transformer station areas simultaneously in the first scenario (broadcast), and to only one meter in the second scenario. The obtained results on 50 sent requests in case of the first scenario are shown in Fig. 8. and Fig. 9. for TSC1 and TSC2, respectively. The results of 50 sent requests in case of the second scenario are depicted in Fig. 10. All results are presented in a histogram form.

In case of the first scenario, MDC sends the request to both TSCs, which forwards a broadcast request to all LMCs and to all 851 meters. All LMCs receive the request simultaneously, download required parameters from meters, according to task priority, and return the required response to TSC. Once responses from all LMCs are gathered, the integral response is transferred from TSC to MDC. As shown in Fig. 8. and Fig. 9. the response time was in most cases below 3.5 minutes, not exceeding 4 minutes in total, in case of the rural type transformer station area (TSC2) with a large number of LMCs.

In case of the second scenario, the response time to almost all requests is less than 10 seconds, except for a few requests where the response time was 15 seconds.

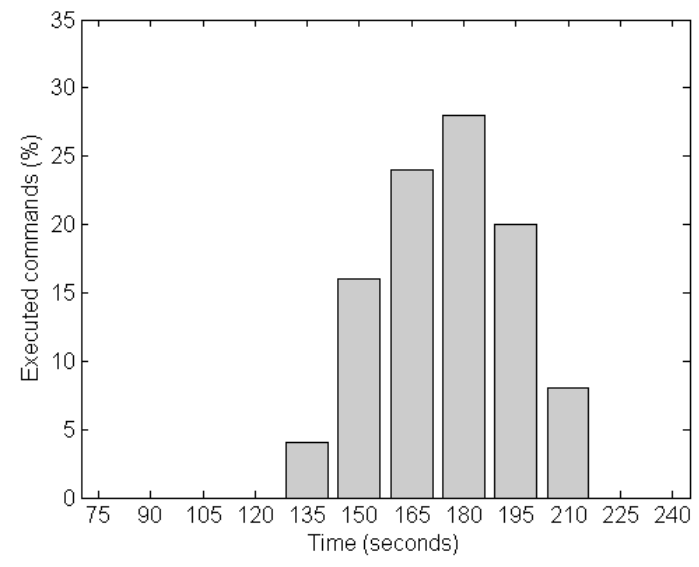

Fig. 8. Broadcast command execution time at MDC layer considering responses of all 448 meters integrated on the TSC 1. 


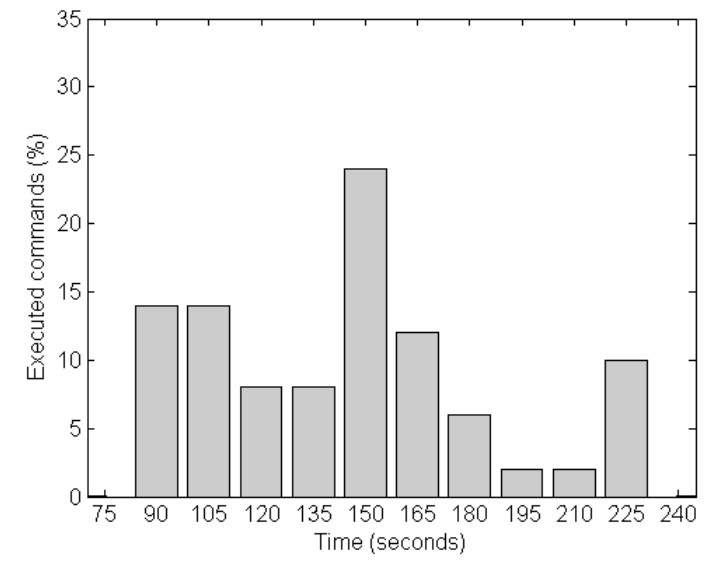

Fig. 9. Broadcast command execution time at MDC layer considering responses of all 403 meters integrated on the TSC 2.

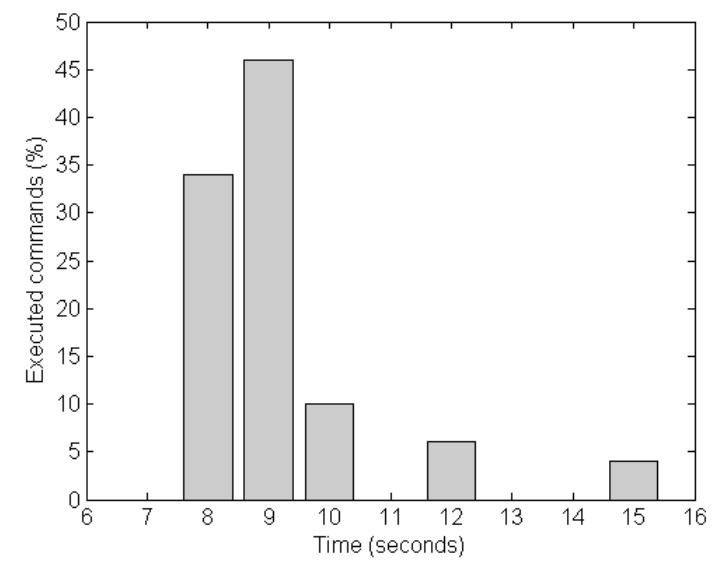

Fig. 10. Single electrical meter command execution time at MDC layer.

\section{CONCLUSION}

The problems of AMI system complexity, information latency, and high communication demands make the traditional architectures of AMI system unable to meet realtime demands, regardless of the communication technology used. The novel layered AMI architecture, proposed in this paper, overcomes aforementioned problems by introducing the new Local Meter Concentrator (LMC) layer. Advantages, compared to traditional metering systems, are presented in the fields of optimized data transfer and introduction of advanced smart metering functionalities, which can be distributed to different layers of the proposed architecture. Nevertheless, the main achieved benefits are real-time data transfer, system interoperability and the possibility to integrate different communication technologies within an AMI system.

Verification results from the presented case study prove the AMI operation has real-time characteristics, reliability, and communication robustness. Further integration of different real-time services and consumer side applications into the proposed architecture will be a part of future work.

\section{ACKNOWLEDGMENT}

The authors are thankful for the support of Unit-EM Ltd. Malta for enabling the access to the smart metering system, making the presented data analyses possible.

\section{REFERENCES}

[1] M. Erol-Kantarci and H. T. Mouftah, "Energy-Efficient Information and Communication Infrastructures in the Smart Grid: A Survey on Interactions and Open Issues," in IEEE Communications Surveys \& Tutorials, vol. 17, no. 1, 2015, pp. 179-197.

[2] Advanced Metering Infrastructure, US Dept. of Energy, Office of Electricity Delivery and Energy Reliability, 2008.

[3] R. Mohassel, A. Fung, F. Mohammadi, K. Raahemifar, "A survey on advanced metering infrastructure and its application in Smart Grids," in Proc. Electrical and Computer Engineering (CCECE), 2014 IEEE 27th Canadian Conference, Toronto, 2014, pp.1-8.

[4] S. Rusitschka, K. Eger and C. Gerdes, "Smart Grid Data Cloud: A Model for Utilizing Cloud Computing in the Smart Grid Domain," in Proc. Smart Grid Communications (SmartGridComm), 2010 First IEEE International Conference, Gaithersburg, 2010, pp. 483-488.

[5] M. Arenas-Martinez, S. Herrero-Lopez, A. Sanchez, J. R. Williams, P. Roth, P. Hofmann and A. Zeier, "A Comparative Study of Data Storage and Processing Architectures for the Smart Grid," in Proc. Smart Grid Communications (SmartGridComm), 2010 First IEEE International Conference, Gaithersburg, 2010, pp. 285-290.

[6] Stephen F. Bush, Smart Grid: Communication-Enabled Intelligence for the Electric Power Grid, Wiley-IEEE Press, 2014.

[7] R. H. Khan and J. Y. Khan, "A comprehensive review of the application characteristics and traffic requirements of a smart grid communications network," Computer Networks, vol. 57, issue 3, 2013, pp. 825-845.

[8] M. Arian, MAPNA group, V. Soleimani, B. Abasgholi, H. Modaghegh and N. S. Gilani, "Advanced Metering Infrastructure System Architecture," in Proc. Power and Energy Engineering Conference (APPEEC), Wuhan, 2011, pp. 1-6.

[9] G. N. Hoang, L. Liquori and H. N. Chan, A Scalable Communication Architecture for Advanced Metering Infrastructure, HAL-Inria, 2013.

[10] Z. Jiazhen, R.Q. Hu and Y. Qian, "Scalable Distributed Communication Architectures to Support Advanced Metering Infrastructure in Smart Grid," IEEE Trans. Parallel and Distributed Systems, vol. 23, issue 9, pp. $1632-1642,2012$.

[11] F. Bouhafs, M. Mackay and M. Merabti, "Links to the Future: Communication Requirements and Challenges in the Smart Grid," IEEE Power and Energy Magazine, vol. 10, issue 1, 2012, pp. 24-32.

[12] European Smart Metering Industry Group, "The potential of smart metering enabled programs to increase energy and systems efficiency: a mass pilot comparison", ESMIG 2011.

[13] I. Petrusevski, M. Zivanovic, A. Rakic, and I. Popovic, "Novel AMI architecture for real-time smart metering," in Proc. Telecommunications Forum Telfor (TELFOR), Belgrade, 2014, pp. 664-667.

[14] Application integration at electric utilities - System interfaces distribution management - Part 9: Interfaces for meter reading and control, IEC 61968-9, Edition 2, 2013.

[15] Electrical metering - Data exchange meter reading, tariff and load control - Part 21: Direct local data exchange, IEC 62056-21, First edition, 200205 .

[16] Electrical metering - Data exchange meter reading, tariff and load control - Part 31: Use of local area networks on twisted pair with carrier signaling, IEC 62056-21, First edition, 1999-11.

[17] S. Gali, A. Scaglione and Z. Wang, "For the Grid and Through the Grid: The Role of Power Line Communications in the Smart Grid," Proceedings of the IEEE, vol. 99, issue 6, 2011, pp. 998 - 1027.

[18] M. Yigit, C. Gungor, G. Tuna, M. Rangoussi, E. Fadel, "Power line communication technologies for smart grid applications: A review of advances and challenges," Computer Networks, vol. 70, 2014, pp. 366383.

[19] N. Bežanić and I. Popović "Service-oriented Sensor Network for Environmental Monitoring," in Proc. Telecommunications Forum Telfor (TELFOR), Belgrade, 2012, pp. 1544 - 1547.

[20] N. Bežanić and I. Popović "Virtual transducers in service-oriented smart transducers network," in Proc. Telecommunications Forum Telfor (TELFOR), Belgrade, 2013, pp. 813-816.

[21] D. Kovačević, A. Rakić, D. Muratović and S. Minić "Smart metering: Implementation Strategy and Example of Practical Application", in Proc. Electrical engineering institute Nikola Tesla, vol. 20, 2010, pp.165-178. 